\title{
Desafios Associados à Formação do Médico em Saúde Coletiva no Curso de Medicina de uma Universidade Pública do Ceará
}

\author{
Challenges Associated with the Formation of \\ the Doctor in Collective Health in the Course of \\ Medicine of a Public University of Ceará
}

\author{
Jéssica Bezerra Custódio \\ Maria das Graças Barbosa Peixoto \\ Carlos André Moura Arruda \\ Daniele Vasconcelos Fernandes Vieira ${ }^{I}$ \\ Maria do Socorro de Sousal \\ Maria Marlene Marques Ávila
}

\section{PALAVRAS-CHAVE}

- Educação Superior.

- Formação Médica.

- Sistema Único de Saúde.

- Saúde Coletiva.

\section{RESUMO}

A implementação do Sistema Único de Saúde (SUS) acarretou a emergência de processos de qualificação de recursos humanos em saúde, o que engendrou a necessidade de construir as concepções curriculares no campo educacional da saúde no Brasil. Assim, o campo da Saúde Coletiva (SC) emerge como profícuo no desenvolvimento da discussão multirreferencial em saúde, no âmbito da educação, formação e trabalho. Este estudo objetivou analisar a percepção de docentes do curso de Medicina de uma universidade pública do Ceará sobre os desafios da formação médica em Saúde Coletiva. Trata-se de um estudo de caso com abordagem qualitativa, realizado com emprego da técnica de grupo focal. Foi conduzida uma sessão de grupo focal com quatro docentes das disciplinas da área da Saúde Coletiva, sendo o material qualitativo organizado em categorias e processado por meio da Análise Temática. Após a análise, emergiram as categorias: preconcepções dos discentes sobre a Saúde Coletiva; infraestrutura e preparação para uso das metodologias ativas; diálogo entre os docentes das disciplinas do eixo de Saúde Coletiva; interlocução entre a teoria e prática médica; papel ativo do discente na aprendizagem; interdisciplinaridade. Os professores assinalaram o preconceito no início do curso, sendo as novas estratégias didático-pedagógicas, pautadas nas metodologias ativas, fundamentais e mais atraentes e adequadas ao contexto social vigente, estimulando a busca do conhecimento e a melhoria da relação aluno-professor. Ademais, reconheceram a existência de fatores específicos do curso de Medicina que dificultam a aplicabilidade de estratégias didático-pedagógicas: fragilidade da formação pedagógica de grande parte do corpo docente para o exercício da docência, devido à inexistência de uma política de educação permanente e à estrutura física inadequada. Ratifica-se a importância das disciplinas da área da Saúde Coletiva para a formação do médico nessa área no que tange às competências propostas pelas DCN 2014, contribuindo para o agir do médico e para sua organização como parte de uma equipe de saúde. Dessa forma, para qualificar a formação médica, inexoravelmente, não se pode prescindir da inserção da Saúde Coletiva na matriz curricular do curso. Para que isso ocorra, é imprescindível que os conteúdos teóricos estejam atrelados ao contexto social e tecnológico vigente e pautados nas metodologias ativas de aprendizagem. 


\section{KEY-WORDS}

- Higher Education.

- Medical Training.

- Unified Health System.

- Collective Health.

Recebido em: 21/9/18

Aceito em: 9/11/18
ABSTRACT

The implementation of the Unified Health System guided the emergence of human resources qualification processes in health, which generated the need to construct the curricular conceptions in the field of health education in Brazil. Thus, the field of Collective Health (SC) emerges as a useful tool in the development of the multireferential discussion on health, in the field of education, training ad work. This study aimed to analyze the perception of teachers of the medical course of a Public University of Ceará on the challenges of medical training in Collective Health. This is a case study with a qualitative approach, based on the focus group technique. A focus group session was held with four professors from the disciplines of the Collective Health area, the qualitative material being organized into categories and processed through the Thematic Analysis. After analysis emerged the categories: students' preconceptions about collective health; infrastructure and preparation for use of active methodologies; dialogue among the teachers of the disciplines of the collective health axis; interlocution between medical theory and practice; active role of the learner in learning; interdisciplinarity. The teachers pointed out the prejudice at the beginning of the course, and the new didactic-pedagogical strategies, based on active methodologies, are fundamental and more attractive and appropriate to the current social context, stimulating the search for knowledge, and improving the student-teacher relationship. In addition, they recognized the existence of specific factors of the medical course that hinder the applicability of didactic-pedagogical strategies: fragility of the pedagogical formation of a large part of the teaching staff for the teaching exercise, due to the inexistence of a Permanent Education Policy and the structure physics. It ratifies the importance of the disciplines of the Collective Health for the training of the doctor in this area with regard to the competencies proposed in the light of the 2014 DCN, corroborating to the physician's action and to his organization as part of a health team. Thus, it is evident that, in order to qualify the medical training, inexorably, one cannot do without the insertion of Collective Health in the Curricular Matrix of the Course. For this to occur, it becomes imperative that the theoretical content be linked to the current social and technological context, based on active learning methodologies.

\section{INTRODUÇÃO}

Desde o reconhecimento da saúde como direito universal e política social a ser assegurado pelo Sistema Único de Saúde (SUS) na Constituição Federal de 1988, o Brasil tem enfrentado vários desafios no caminho para a efetivação desta conquista. Na formação dos profissionais de nível superior, muitas têm sido as iniciativas de estímulo às mudanças, mas tem se perpetuado nas instituições formadoras o conservadorismo, que não promove a formação adequada de novos perfis de profissionais para o SUS e para a qualificação da gestão e do controle social, como deveria ser ${ }^{1}$.

Neste sentido, apesar dos avanços nos currículos dos cursos de Medicina dos últimos anos, no que tange à abrangência em Saúde Coletiva, percebe-se a necessidade de maior aprofundamento nas discussões e estudos na área de formação e educação médica, sobretudo no que diz respeito à atuação em contextos interdisciplinares e que atravessam diretamente o campo da Saúde Coletiva ${ }^{2}$.

Esta necessidade vem sendo pontuada de forma mais intensa desde as Diretrizes Curriculares Nacionais (DCN) de $2001^{3}$, quando os eixos estruturantes da Saúde Coletiva - epidemiologia, ciências sociais, planejamento e gestão - foram incluídos nas competências a serem desenvolvidas na formação médica. Entretanto, uma pesquisa ${ }^{4}$ avaliou, na perspectiva dos estudantes, a Saúde Coletiva no conjunto das disciplinas, concluindo que ainda existe urgência em repensar a reformulação curricular neste campo e mudar as atitudes docentes. As DCN mais recentes de 2014 aumentaram a ênfase na Saúde Coletiva, dando mais um enfoque a essa necessidade de mudança curricular ao trazer três áreas de competências - Atenção à Saúde, Gestão em Saúde e Educação em Saúde - como estruturantes da formação médica ${ }^{3}$. 
Destarte, é de extrema relevância que haja constante avaliação da formação em saúde e, por conseguinte, da formação em Medicina nas escolas públicas brasileiras, uma vez que há o compromisso de formar recursos humanos para a atuação no SUS condizentes com as necessidades de saúde emergentes.

Desse modo, o presente estudo visa responder à seguinte questão de pesquisa: "Quais são os desafios associados à formação médica em Saúde Coletiva na percepção de docentes de uma universidade pública do Ceará?". Assim, este artigo objetiva analisar as percepções de docentes do curso de Medicina de uma universidade pública do Ceará sobre os desafios da formação médica em Saúde Coletiva.

\section{METODOLOGIA}

Trata-se de um estudo de caso com abordagem qualitativa, realizado com emprego da técnica de grupo focal. O estudo de caso contribui para uma visão abrangente de acontecimentos da vida real, constituindo uma importante estratégia metodológica para a pesquisa em ciências humanas, por possibilitar ao investigador o aprofundamento em relação ao fenômeno estudado, permitindo seu amplo e detalhado conhecimento ${ }^{5}$. Por sua vez, a escolha da técnica de grupo focal se justifica pela possibilidade de subsidiar a coleta de dados marcada pela subjetividade de interlocução e a análise das discussões em grupo ${ }^{6}$.

A pesquisa foi realizada no curso de graduação em Medicina de uma universidade pública do Estado do Ceará no período de novembro de 2016 a julho de 2017. O curso tem como missão promover e manter a saúde individual e coletiva da população brasileira, e cearense em particular, por meio de formação médica científica, ética e humanista. A área da Saúde Coletiva é parte integrante da matriz curricular deste curso desde sua criação em 2002, sendo composta pelas disciplinas: Introdução à Saúde Coletiva; Educação em Saúde; Ciências Sociais; Epidemiologia; Políticas Públicas de Saúde; Planejamento em Saúde; Informação e Avaliação em Saúde, ministradas nos três primeiros anos do curso.

A seleção dos participantes da pesquisa foi realizada por meio da lista dos professores por disciplina no projeto pedagógico do curso (PPC) ${ }^{7}$, disponibilizada pela Coordenação do curso. Os participantes foram recrutados mediante convite verbal. Foram incluídos como sujeitos da pesquisa oito docentes responsáveis pelas disciplinas da área da Saúde Coletiva no período do estudo e excluídos dois docentes por serem autores da presente pesquisa. Entretanto, dois professores não compareceram por intercorrências particulares, sendo o grupo final composto por quatro participantes.

O grupo focal (GF) foi realizado em dia e horário mais conveniente para os docentes, sendo conduzido por meio de um roteiro que possibilitou explorar: a percepção sobre a aplicação dos saberes da Saúde Coletiva na prática médica e como eles o faziam; se identificavam a necessidade de reorganização curricular nas disciplinas da área para atender às DCN de 2014; e quais metodologias de ensino-aprendizagem eles consideravam mais apropriadas ao alcance deste propósito.

O GF teve duração de uma hora e trinta minutos e foi conduzido em sala privativa por um moderador da equipe de pesquisadores. Também houve a participação de outra pesquisadora, que atuou como observadora. Toda a sessão do grupo focal foi gravada por meio de um gravador de voz mediante prévio consentimento dos participantes e, posteriormente, transcrita. Além disso, a observadora do grupo focal utilizou um diário de campo para registro escrito da operacionalização do grupo, bem como das reações e interações entre os participantes

O GF foi orientado por um roteiro norteador que continha perguntas disparadoras acerca do objeto em análise: "Vocês reconhecem possibilidades de aplicação dos conhecimentos ministrados na área da Saúde Coletiva na prática médica? Como isso se materializa? Vocês identificam a necessidade de organizar os conhecimentos curriculares nas disciplinas da área da Saúde Coletiva para atender às DCN de 2014? Quais metodologias de ensino-aprendizagem vocês consideram mais apropriadas para organizar este corpo de conhecimento?". O moderador ia lançando tais perguntas, e os participantes, à medida que se sentiam instigados, as respondiam com base em suas vivências práticas nas disciplinas por eles conduzidas. As perguntas norteadoras iam sendo ressignificadas e aprofundadas a partir do discurso dos informantes. $\mathrm{O}$ moderador e o observador, ao final do grupo, elaboraram um diário de campo que subsidiou a organização das categorias temáticas.

Após a transcrição das informações obtidas no grupo focal, foi empregada a técnica de análise temática ${ }^{8,9}$, sendo realizadas pré-análise, exploração do material, tratamento dos resultados obtidos e interpretação. Na pré-análise, o material qualitativo foi ordenado seguindo procedimentos que incluem a leitura exaustiva e a elaboração de um $\operatorname{corpus}{ }^{10}$ que contemplou as percepções explicitadas pelos informantes. Após essa etapa, foi possível identificar temáticas centrais que agregaram dimensões distintas dos depoimentos e que constituíram as categorias analíticas: preconcepções da saúde coletiva pelos discentes; papel ativo do discente na aprendizagem; infraestrutura e preparação para uso das metodologias ativas; diálogo entre os docentes das disciplinas do eixo de Saúde Coletiva; interlocução entre a teoria e prática médica; interdisciplinaridade. 
Nos depoimentos ilustrativos dos resultados, os informantes foram identificados pela letra "P" (abreviação de professor), seguida por um número conforme sua intervenção no GF.

A pesquisa cumpriu todas as exigências da Resolução $n^{\text {o }}$ 466/2012 do Conselho Nacional de Saúde (CNS) ${ }^{11}$ e foi aprovada pelo Comitê de Ética em Pesquisa, sob o Parecer $\mathrm{n}^{\mathrm{o}}$ $388.536 / 2013$. Todos os participantes foram informados sobre os objetivos e métodos da pesquisa, e a participação deles foi condicionada à assinatura do Termo de Consentimento Livre e Esclarecido (TCLE), assegurando-se o sigilo e a privacidade das informações obtidas.

\section{RESULTADOS E DISCUSSÃO}

Preconcepções dos discentes sobre a Saúde Coletiva

Um dos desafios mencionados pelos docentes foi contribuir com a reelaboração de preconcepções dos discentes em relação ao sentido da Saúde Coletiva, para o entendimento do todo social, biológico e ambiental em que se baseiam as suas ações:

[...] a gente trabalha inclusive com algumas preconcepções que os próprios estudantes já trazem, do primeiro semestre, inclusive: de que Saúde Coletiva só é atenção primária, de que Saúde Coletiva não é importante. Então assim, acho que o primeiro passo que a gente dá enquanto docente é desconstruir alguns desses conceitos, na verdade, reelaborar alguns desses conceitos [...] (P1)

O processo de ensino-aprendizagem da área da Saúde Coletiva na instituição investigada ocorre nos três primeiros anos do curso, período de forte concentração de disciplinas de ciências morfológicas, ciências fisiológicas, anatomia aplicada, em que os discentes têm aproximações incipientes com os serviços de Atenção Primária à Saúde, com pouco conhecimento da realidade social que determina os processos de adoecimento e morte da população. Com efeito, alguns docentes, com o propósito de contribuir para a construção de um pensamento reflexivo e próximo da realidade biopsicossocial, adotam estratégias pedagógicas para inserção dos discentes, desde o primeiro semestre letivo, nos cenários de prática, por meio de visitas técnicas nos diversos territórios de saúde, projetos de extensão universitária, como o Programa de Educação pelo Trabalho para a Saúde (PET-Saúde), além de mesas-redondas, painéis e seminários, contando com a participação de profissionais dos serviços de saúde.

Acerca desse aspecto, as Diretrizes Curriculares Nacionais de 2014, em sua parte VII da seção II, abordam a impor- tância da construção participativa do sistema de saúde, pois isto permitirá a adequada elaboração da política de saúde brasileira ${ }^{3}$. Assim, há uma grande necessidade de que os docentes do ensino superior aprimorem competências profissionais para subsidiar uma formação crítica social. É preciso, portanto, substituir as formas tradicionais por metodologias ativas de aprendizagem ${ }^{12}$

\section{Papel ativo do discente na aprendizagem}

A pluralidade de estratégias didático-pedagógicas foi posta em evidência, enfatizando-se a necessidade de utilizar variadas estratégias para estimular o papel ativo do discente na aprendizagem para facilitar a assimilação dos conteúdos:
As nossas estratégias didático-pedagógicas devem contem- plar cada vez mais uma pluralidade [...], até porque existem inteligências múltiplas, [...], quanto mais a gente conseguir protagonizar o estudante, seria fundamental que ele pudesse construir o conhecimento, e esse conhecimento construído coletivamente. (P1)

\section{[...] partindo dessa realidade concreta, tem uma estratégia que [...] eu não utilizo muito mas gostaria de utilizar mais, que é a aula de campo, a qual você vai para dentro de uma co- munidade [...] e a partir dessa realidade a gente faz discussões [...] apontando para o protagonismo. (P2)}

De acordo com as DCN de 2014, no quesito da promoção da construção e socialização do conhecimento, evidencia-se a necessidade de os docentes serem abertos à transformação do conhecimento e da própria prática, com escolha de distintas estratégias interativas para a construção de conhecimentos ${ }^{3}$.

A problematização, os seminários, os trabalhos em pequenos grupos, os relatos críticos de experiências e as mesas-redondas, por exemplo, são estratégias utilizadas pelas metodologias ativas como recursos didáticos de ensino-aprendizagem, objetivando alcançar e motivar o estudante a ressignificar suas descobertas, facilitando a fixação de conteúdos ${ }^{13}$.

Todas essas metodologias ativas, portanto, fazem parte, intrinsecamente, das categorias de avaliação dos estudantes segundo as Diretrizes Curriculares Nacionais de 2014, conforme o artigo 31, que estabelece que as avaliações dos estudantes se basearão em conhecimentos, habilidades, atitudes e conteúdos curriculares desenvolvidos ${ }^{3}$.

No modelo pedagógico norteado pelo PBL, por exemplo, o foco do processo educativo está centrado no estudante e baseado na resolução de problemáticas, com estímulo à autoformação discente ${ }^{12}$. Isto se assemelha bastante a cinco itens (I a 
V) da seção II das DCN de 2014, que consistem na necessidade de aprender a aprender como parte do processo de ensino-aprendizagem; em ter autonomia e perceber a necessidade da educação continuada; em aprender com a troca de saberes multiprofissionais; em aprender em situações controladas, como simulações de realidade; e em comprometer-se com o processo de formação ${ }^{3}$.

Além disso, no Artigo 32 das Diretrizes Nacionais Curriculares de 2014, consta que o curso de graduação em Medicina deverá utilizar metodologias ativas e critérios para acompanhamento e avaliação do processo ensino-aprendizagem, uma vez que a eficácia depende da qualidade da moderação e da experiência do professor na aplicabilidade da estratégia em questão ${ }^{3}$.

Segundo Morán ${ }^{12}$, hoje as instituições educacionais escolhem geralmente entre dois caminhos: um menos radical, com mudanças progressivas, e outro mais amplo, com mudanças profundas. O primeiro mantém o modelo curricular tradicional, com disciplinas, mas prioriza a participação mais ativa do aluno, com metodologias ativas, como o ensino por projeto, de forma mais interdisciplinar, o ensino híbrido ou blended e a sala de aula invertida. Outras instituições propõem modelos mais bruscos de transformação curricular, disruptivos, que redesenham o projeto e os espaços físicos, baseando-os em atividades em que cada aluno aprende com os outros em grupos e projetos, com supervisão de professores orientadores.

Assim, torna-se muito mais fácil para as instituições que conseguem se adequar a esses projetos pedagógicos transformados, seja suave ou radicalmente, o alcance dos princípios das DCN de 2014, sendo, portanto, uma dificuldade evidentemente exposta no cenário estudado.

\section{Infraestrutura e preparação para uso das metodologias ativas}

Em alguns depoimentos, nota-se que nem todos os docentes estão capacitados a aplicar novas estratégias didático-pedagógicas, além de a instituição não oferecer infraestrutura adequada para a aplicação das metodologias ativas de aprendizagem:

[...] a gente tem dificuldade de realizar, porque além desse preparo docente, tem a questão de estrutura também [...] como é que eu vou induzir esse protagonismo, essas dinâmicas de grupo, se eu não tenho uma estrutura adequada ou eu mesmo professor não tenho preparo para conduzir esse tipo de estratégia? (P1)

[...] identificamos claramente a necessidade de mudanças metodológicas e educacionais. No meu caso, seria construir um laboratório de habilidades, para falar de habilidades comunicacionais, em que teríamos que construir espaço e infraestrutura que permitam construir situações que podem ser gravadas e discutidas com os alunos, que outras universidades têm e que a nossa não tem. (P2)

Apesar da intenção dos docentes de elaborar estratégias didático-pedagógicas de atingir os objetivos das DCN 2014, há a barreira estrutural específica da universidade, que restringe a atuação do professor em sala de aula e dificulta o processo de ensino-aprendizagem. Essa situação desmotiva estudantes e professores a buscarem novas estratégias didático-pedagógicas que melhorem a qualidade da formação e dificulta a realização de grupos de estudos dentro da universidade ${ }^{14}$.

A sala de aula é o principal espaço em que são trabalhadas as relações iniciais entre discente e professor. $\mathrm{O}$ processo de ensino-aprendizagem é um objetivo complexo, que depende de muitas variáveis além do estudante e do professor. Se não houver inicialmente um ambiente cômodo de estudo coletivo, o processo de ensino ficará defasado. Assim, as instalações e materiais institucionais são fatores que podem interferir na aprendizagem dos estudantes ${ }^{15}$.

Frente aos comentários sobre as dificuldades estruturais do curso de Medicina estudado, nota-se que, para que as mudanças dos métodos de ensino-aprendizagem aconteçam, são necessárias transformações estruturais para que determinada metodologia ativa adaptada à realidade da instituição seja capaz de direcionar a organização curricular ${ }^{13,16}$.

Outra dificuldade estrutural apresentada pelos docentes consiste em biblioteca desatualizada e sem estrutura adequada às propostas que as metodologias ativas exigem:

\section{[...] o problema é que falta uma biblioteca [...]. Como é que você faz um processo de ensino-aprendizagem em uma uni- versidade sem uma biblioteca? Ou você entra numa lógica chamada de "pré-cozido", em que cada professor já prepara seu material xerocado, ou não dá certo. Esse é um outro ele- mento que matou completamente a possibilidade ou recurso de PBL. (P2)}

Percebe-se que o ambiente físico tanto das salas de aulas como da escola como um todo, incluindo bibliotecas, precisaria ser redesenhado segundo uma concepção mais ativa. Assim, a biblioteca é um dos agentes estruturais necessários para maior facilidade de implantação de metodologias pautadas na maior participação do aluno, o que está presente na universidade em questão, porém necessita de reformas significativas de estrutura e conteúdo. Diante de tais desafios, destaca-se a 
importância de a universidade organizar oficinas de atualização em estratégias didático-pedagógicas que favoreçam a autoaprendizagem ${ }^{17}$.

\section{Diálogo entre os docentes das disciplinas do eixo de Saúde Coletiva}

Um dos desafios mencionados pelos doentes foi o diálogo entre os professores para planejamento compartilhado das disciplinas da área de Saúde Coletiva, de maneira a evitar a repetição de conteúdos:

É um desafio bem complicado de se realizar, que é essa comunicação entre os professores da Saúde Coletiva [...] de saber o que cada um ministra, para que a gente não vá falar novamente o que o outro professor já falou, para tentar ver o lado dos alunos, pois às vezes o que eles falam muitas vezes de fato acontece, de fato estamos repetindo conteúdo. (P1)

E aí o que eu acho que está faltando, é justamente essa integração, que eu acho que a gente tem que sentar, conversar e ver, até porque um discurso que os alunos da Medicina usam muito, principalmente na nossa área, é a questão de que os conteúdos são repetidos. (P4)

Nesses depoimentos, há de se reconhecer que a reestruturação curricular envolve repensar a organização do conhecimento na prática. Para isso, é essencial priorizar uma educação sustentada na interdisciplinaridade, articulando os três eixos de formação de modo integral (ensino, pesquisa e extensão), cada vez mais de acordo também com os princípios e diretrizes do SUS ${ }^{18}$. Para alcançarem essa interdisciplinaridade, os docentes precisam trabalhar juntos e articular seus conhecimentos de forma complementar e organizada, de modo a buscar a participação ativa dos discentes no processo de ensino.

A fragmentação dos conteúdos presentes na matriz curricular e as consequências que isso acarreta são um dos reflexos da falta de comunicação entre os professores ${ }^{4}$. Um estudo realizado em 2011 com 129 estudantes regularmente matriculados do primeiro ao décimo segundo semestre do curso de graduação em Medicina da Universidade Estadual do Ceará concluiu que os estudantes, frente às disciplinas da Saúde Coletiva, têm como principais desejos de mudanças a fusão de várias disciplinas com o mesmo conteúdo programático $(47,7 \%)$ e maior comunicação entre os professores $(26,6 \%)$. Tal estudo representa o posicionamento geral dos estudantes da instituição, que demonstra a necessidade de atenção para que as técnicas de ensino-aprendizagem atualmente utilizadas se transformem como um todo a fim de que os discentes alcancem os objetivos de aprendizagem de cada disciplina de modo uniforme e integrado ${ }^{4}$.

\section{Interlocução entre a teoria e a prática médica}

Na perspectiva de tornar o ensino da Saúde Coletiva mais atraente para os discentes de Medicina, os docentes reconhecem a necessidade do uso de metodologias que incentivem a participação do estudante mediante a abordagem de problemas e situações reais:

Nas disciplinas que eu ministro, eu tento o máximo que posso mostrar a aplicabilidade na prática médica, e, assim, não só do ponto de vista de uma prática médica relacionada a má gestão dos serviços de saúde [...], mas também até a aplicabilidade na clínica. (P1)

[...] todas as aulas têm uma parte introdutória, mas têm trabalho de grupo a partir de casos clínicos que os alunos tentam solucionar, discutir [...], mas também grupo de role play, jogo de papéis, um faz o papel de médico, o outro de enfermeiro, e o outro de observador, então tem sempre uma dimensão prática a partir de casos ao longo do percurso. (P2)

[...] a gente sempre tenta contextualizar todos os conceitos, porque a nossa disciplina, os alunos dizem que é muito conceito, é muita "decoreba", mas a gente tenta passar longe dessa questão, porque a gente tenta colocar na cabeça dos alunos a lógica por trás de tudo que a gente apresenta na disciplina, e também contextualizando as questões dos conceitos a partir de exemplos. (P4)

A preocupação dos discentes em não dissociar a teoria da prática demonstra a fuga dos moldes conservadores, limitados à dimensão biológica e individual, que já se mostrou ineficaz em termos de aplicabilidade à realidade atual ${ }^{19}$. Deve-se, então, contestar progressivamente a concepção do currículo normativo, na qual o discente detém os conhecimentos de forma alheia à realidade e a sua aplicação na prática médica.

A estratégia de role play possibilita a dramatização interpessoal, aproximando o acadêmico do real mesmo distante do cenário prático, de modo a sensibilizar cada ator acerca da importância de cada função. Isto capacita o acadêmico a desenvolver confiança nas ações executadas e a reconhecer o papel que a pessoa exerce na relação médico-paciente ${ }^{20}$.

Um estudo com o objetivo de analisar a formação médica na percepção dos estudantes e professores identificou que, na percepção desses sujeitos, a metodologia adotada é muito tra- 
dicional e fragmentada, sem estimular o pensamento crítico ou propor inter-relações entre teoria e prática ${ }^{14}$

\section{Interdisciplinaridade}

Neste estudo, a interdisciplinaridade foi outro desafio mencionado pelos docentes no ensino da Saúde Coletiva:

[...] necessidade de trabalhar e ter interdisciplinaridade, inclusive até em alguns momentos a transdisciplinaridade [...] (P1)

[...] então nosso esforço no futuro deve seguir uma lógica interdisciplinar, e ao ver como as disciplinas dialogam entre si, e tentar construir formas de pensamento, que conjugam conhecimentos e práticas que vêm de múltiplas áreas. (P2)

É importante destacar que, segundo Peduzzi et al. ${ }^{21}$, "a tendência dos profissionais de cada área trabalhar de forma isolada e independente das demais expressa sua longa e intensa formação também isolada e circunscrita à sua própria área de atuação".

No parágrafo IV do Artigo 29 das DCN de 2014, consta a necessidade de promover a integração e a interdisciplinaridade em coerência com o eixo de desenvolvimento curricular, buscando unir as diversas dimensões de atuação do indivíduo e promover a percepção holística do médico em formação. Além disso, a estrutura do curso de graduação em Medicina deve utilizar metodologias que priorizem a participação ativa do aluno na formação do conhecimento e na integração entre os conteúdos ${ }^{3}$. Outro aspecto observado é que o ensino nos moldes da interdisciplinaridade fortalece o trabalho em equipe, transformando as práticas de saúde com foco nas necessidades de saúde da população ${ }^{21}$.

Um estudo sobre vivências de acadêmicos de Medicina durante a formação médica apontou lacunas principalmente em relação a atividades práticas que incluam atenção integral e interdisciplinar. A formação para a interdisciplinaridade foi considerada incipiente, sendo pouco comuns as atividades que contemplaram vivência interdisciplinar, o que compromete o desenvolvimento de uma atenção integral em saúde ${ }^{22}$.

\section{CONSIDERAÇÕES FINAIS}

Na percepção dos discentes, entre os desafios associados à formação médica em Saúde Coletiva estão: preconcepções dos discentes acerca da Saúde Coletiva; infraestrutura e preparação para uso das metodologias ativas; diálogo entre os docentes das disciplinas do eixo de Saúde Coletiva; interlocução entre a teoria e a prática médica; papel ativo do discente na aprendizagem; e interdisciplinaridade.
Para qualificar a formação médica na área da Saúde Coletiva, é imprescindível que os conteúdos teóricos estejam atrelados ao contexto social e tecnológico vigente e pautados nas metodologias ativas de aprendizagem, com estímulo ao protagonismo dos discentes na aprendizagem. Nesse sentido, essas estratégias seriam fundamentais para desenvolver caminhos no ensino da Saúde Coletiva de maneira mais atraente.

A situação no curso de Medicina estudado, em meio ao cenário de importância da necessidade de utilização das metodologias ativas no processo de ensino-aprendizagem, evidencia o despreparo do corpo docente para aplicar métodos que proporcionem maior envolvimento do discente na sala de aula. A estrutura física inadequada foi também mencionada como um fator limitante para o uso de metodologias ativas.

A inexistência de uma política de educação permanente para os docentes da referida instituição repercute negativamente no processo formativo. Assim, é importante a qualificação do processo de ensino-aprendizagem, por meio do desenvolvimento da capacidade de utilização de estratégias cooperativas de aprendizagem, numa perspectiva de relação horizontal de confiança com os discentes.

\section{REFERÊNCIAS}

1. Ceccim RB, Feuerwerker LCM. O Quadrilátero da Formação para a Área da Saúde: Ensino, Gestão, Atenção e Controle Social. Physis: Revista de Saúde Coletiva 2014;14(1):41-65.

2. Medeiros MAT, Braga-Campos FC, Moreira MIB. A integralidade como eixo da formação em proposta interdisciplinar: estágios de Nutrição e Psicologia no campo da Saúde Coletiva. Rev. Nutr. Campinas 2014;27(6):785-98.

3. Brasil. Ministério da Educação. Conselho Nacional de Educação. Câmara de Educação Superior. Resolução CNE/CES 3/2014 no 3 de 20 de junho de 2014. Institui Diretrizes Curriculares do Curso de Graduação em Medicina e dá outras providências. Diário Oficial da União. Brasília, 23 de junho de 2014; Seção 1; p. 8-11.

4. Oliveira JAA, Jorge MSB, Silva MGC, Pinto DM, Ointo FJM. A Saúde Coletiva na Formação dos Discentes do Curso de Medicina da Universidade Estadual do Ceará. RevBras de EducMéd 2011;35(3):398-404.

5. YIN RK. Estudo de caso: planejamento e métodos. 5 ed., Porto Alegre: Bookman; 2015.

6. Arantes PCC, Deusdará B. Grupo focal e prática de pesquisa em análise do discurso: metodologia em perspectiva dialógica. Rev. de Estudo da Linguagem. Belo Horizonte, 2017;25(2):792-814. 
7. Universidade Estadual do Ceará. Curso de Medicina. Projeto Político-Pedagógico do Curso de Medicina. UECE, 2012.

8. Minayo MCCS, Assis SG, Souza ER. Avaliação por Triangulação de Métodos: Abordagem de Programas Sociais. Cad Saúde Pública 2016;22(5):1115-8.

9. Bardin L. Análise de conteúdo. Tradução: Luís Antero Reto e Augusto Pinheiro. São Paulo: Edições 70, 2011.

10. Dal-Farra, RAP; Lopes, TC. Métodos Mistos de Pesquisa em Educação: pressupostos teóricos. Nuances: estudos sobre Educação. Presidente Prudente-SP, 2013;24(3):67-80.

11. Brasil. Ministério da Saúde. Resolução no ${ }^{\circ}$ 466, de 12 de dezembro de 2012. Conselho Nacional de Saúde. Diário Oficial [da] República Federativa do Brasil. 2013, junho, 13; Seção 1. p. 59-62.

12. Morán J. Mudando a Educação com Metodologias Ativas. Rev. Convergências Midiáticas, Educação e Cidadania: aproximações jovens 2015; 2:17.

13. Paiva MRF, Parente JRF, Brandão IR, Queiroz AHB. Metodologias ativas de ensino-aprendizagem: Revisão integrativa. SANARE 2016;15(2):145-153.

14. Chagas WEC. O ensino Médico no Ceará: o caso do curso de Medicina da UECE. Fortaleza; 2016. Mestrado (Dissertação) - Curso de Mestrado Profissional Ensino na Saúde, Universidade Estadual do Ceará.

15. Borges MC, Chachá SGF, Quintana SM, Freitas LCL, Rodrigues MLV. Aprendizado Baseado em Problemas. Rev. FMRP 2014; 47(3):301-7.

16. Almeida EG, Batista NA. Desempenho Docente no Contexto PBL: Essência para Aprendizagem e Formação Médica. RevBras de EducMéd 2013; 37(2):192-201.

17. Monteiro JS, Silva DP. A Influência da Estrutura Escolar no processo de ensino-aprendizagem: uma análise baseada nas experiências do estágio supervisionado em Geografia. Rev. Geografia Ensino e Pesquisa 2015;19(3):19-28.

18. Medeiros MAT, Braga-Campos FC, Moreira MIB. A Integralidade como eixo da formação em proposta interdisciplinar: estágios de Nutrição e Psicologia no campo da Saúde Coletiva. Rev. Nutrição, Campinas 2014; 27(6):785-798.
19. Bezerra AF, Meneses LBA, Carvalho OS, Soares VL, Meneses LBA, Soares MJOG. Currículo dos Cursos de Saúde sob a Óptica dos Docentes. Rev enferm UFPE 2015;9(11):9723-32.

20. Cogo ALP, Pai DD, Aliti GB, Hoefel HK, Azzolin KO, Busin L, Unicovsky MAR, Kruse MHL. Casos de Papel e Role Play: estratégias de aprendizagem em enfermagem. Rev. Brasileira de Enfermagem 2016; 69(6):1231-5.

21. Peduzzi M, Norman IJ, Germani ACCG, Silva, JAM, Souza GC.Educação interprofissional: formação de profissionais de saúde para o trabalho em equipe com foco nos usuários. RevEscEnferm USP2013;47(4):977-83.

22. Santos RNLC, Ribeiro, KSQS, Anjos UU, Farias DN, Lucena EMF. Integralidade e Interdisciplinaridade na Formação de Estudantes de Medicina. RevBras de EducMéd 2015; 39(3):378-387.

\section{CONTRIBUIÇÃO DOS AUTORES}

Maria das Graças Barbosa Peixoto: orientação da pesquisa, participou da concepção, desenho do estudo, análise e interpretação dos dados, discussão e elaboração do artigo. Jéssica Bezerra Custódio: coleta de dados, análise e interpretação dos dados e elaboração do artigo. Carlos André Moura Arruda: facilitação do grupo focal, análise dos dados e revisão final do artigo. Daniele Vasconcelos Fernandes Vieira: revisão final do artigo. Maria do Socorro de Sousa: revisão teórico-metodológica e final do artigo. Maria Marlene Marques Ávila: revisão teórico-metodológica e final do artigo.

\section{CONFLITO DE INTERESSES}

Os autores declaram não haver conflito de interesses.

\section{ENDEREÇO PARA CORRESPONDÊNCIA}

Maria das Graças Barbosa Peixoto

Av. Rogaciano Leite, №200; Bloco Azalee; Apto.1301

Salinas - Fortaleza, Ceará

CEP: 60810786

E-mail: maria.gracas@uece.br 